\title{
ARTICLE Burst activation of dopamine neurons produces prolonged post-burst availability of actively released dopamine
}

\author{
Sweyta Lohani ${ }^{1}$, Adria K. Martig ${ }^{1,4}$, Suzanne M. Underhill ${ }^{2,5}$, Alicia DeFrancesco ${ }^{1,6}$, Melanie J. Roberts ${ }^{1}$, Linda Rinaman ${ }^{1,7}$,
} Susan Amara ${ }^{2,5}$ and Bita Moghaddam ${ }^{1,3}$

Both phasic and tonic modes of neurotransmission are implicated in critical functions assigned to dopamine. In learning, for example, sub-second phasic responses of ventral tegmental area (VTA) dopamine neurons to salient events serve as teaching signals, but learning is also interrupted by dopamine antagonists administered minutes after training. Our findings bridge the multiple timescales of dopamine neurotransmission by demonstrating that burst stimulation of VTA dopamine neurons produces a prolonged post-burst increase (>20 min) of extracellular dopamine in nucleus accumbens and prefrontal cortex. This elevation is not due to spillover from the stimulation surge but depends on impulse flow-mediated dopamine release. We identified Rhomediated internalization of dopamine transporter as a mechanism responsible for prolonged availability of actively released dopamine. Thus, a critical consequence of burst activity of dopamine neurons may be post-burst sustained elevation of extracellular dopamine in terminal regions via an intracellular mechanism that promotes dopamine transporter internalization. These results demonstrate that phasic and tonic dopamine neurotransmission can be a continuum and may explain why both modes of signaling are critical for motivational and cognitive functions associated with dopamine.

Neuropsychopharmacology (2018) 43:2083-2092; https://doi.org/10.1038/s41386-018-0088-7

\section{INTRODUCTION}

Dopamine neurons project extensively to striatal and cortical regions where they mediate a diverse array of functions, including reward-related learning, drug-related synaptic plasticity, working memory, and motivation [1-6]. Dopamine neurons respond with brief and phasic bursts of action potentials to salient events, such as reward-predictive stimuli and unexpected reward delivery $[7,8]$, and these bursts cause phasic dopamine release in terminal regions, including the prefrontal cortex (PFC) and the nucleus accumbens (NAC) [9-12]. Influential theories suggest these phasic responses serve as teaching signals in reinforcement learning $[8$, $13,14]$ and/or motivational signals that mediate cue-triggered seeking of rewards $[4,6]$.

While fast-scan cyclic voltammetry (FSCV) studies have characterized rapid and transient increases in extracellular dopamine concentration $\left([D A]_{0}\right)$ in response to salient events in a behavioral task [15-18], consistent with phasic bursting of dopamine neurons, microdialysis studies have demonstrated sustained increases in cortical and striatal extracellular dopamine that persist for many minutes (even up to an hour) after the completion of appetitive learning, instrumental behavior, and other cognitive tasks [19-23]. The prolonged post-session dopamine increase occurs even though animals are no longer receiving rewards and/or being presented with salient stimuli.
Importantly, dopamine antagonists administered either systemically or directly into PFC and striatum minutes after behavioral training can disrupt memory consolidation, demonstrating the necessity of post-training dopamine signaling [24-26]. This slower mode of dopamine signaling has been attributed to the so-called "tonic" dopamine release, which is hypothesized to be mediated by mechanisms that are entirely distinct from those that drive phasic activity of dopamine neurons and release [27-29]. But given that learning and most behavioral functions assigned to dopamine depend on both fast and slow modes of neurotransmission, we hypothesized that these phasic and tonic dopamine states might instead comprise a continuum of the same activation process in an awake and active animal. In that case, phasic activation of dopamine neurons could trigger secondary events that enhance the slow (tonic) activation of dopamine levels. Thus, we designed experiments to determine whether bursting of dopamine neurons results in post-burst sustained elevation of extracellular levels of dopamine in terminal regions and, if so, what mechanisms sustain this elevation.

Dopamine's effects on reward-related learning, motivation, and cognitive constructs such as attention and working memory are primarily mediated by dopamine neurons in ventral tegmental area (VTA) and their projections to the NAC and PFC $[2,5,30-32]$. Thus, we assessed $[D A]_{0}$ in these two terminal regions before and

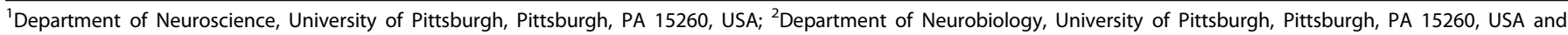
${ }^{3}$ Department of Behavioral Neuroscience, Oregon Health and Science University, Portland, OR 97239, USA

Correspondence: Bita Moghaddam (bita@ohsu.edu)

${ }^{4}$ Present address: The New York Academy of Sciences, New York, NY, USA

${ }^{5}$ Present address: National Institute of Mental Health, Bethesda, MD, USA

${ }^{6}$ Present address: Case Western Reserve University, Cleveland, $\mathrm{OH}$, USA

${ }^{7}$ Present address: Department of Psychology, Florida State University, Tallahassee, FL, USA

These authors contributed equally: Sweyta Lohani, Adria K. Martig.
}

Received: 17 December 2017 Revised: 26 April 2018 Accepted: 29 April 2018

Published online: 7 May 2018 
after activation of VTA neurons. We found that electrical and optogenetic stimulation of VTA neurons, using patterns of activation that resemble the phasic bursting activity of dopamine neurons during reward-guided behavioral tasks, results in increased $[D A]_{0}$ in the NAc and PFC that persists for many minutes after stimulation is terminated. This sustained poststimulus elevation of dopamine is blocked by tetrodotoxin (TTX), suggesting that it is not due to dopamine spillover from the initial stimulation; rather, it results from reduced dopamine transporter (DAT)-mediated clearance of dopamine released spontaneously during the post-stimulation period.

\section{MATERIALS AND METHODS}

Adult male Sprague-Dawley or Long-Evans rats were used. All experiments were approved by and conducted according to the guidelines and regulations of the Institutional Animal Care and Use Committee at the University of Pittsburgh and the $\mathrm{NIH}$.

Briefly, for microdialysis experiments, animals were implanted with microdialysis probes in the PFC and/or NAc and bipolarstimulating electrodes (electrical stimulation) or optical fibers (optogenetic stimulation) in the VTA. Microdialysis was conducted in freely moving animals, and dialysate samples were collected every $20 \mathrm{~min}$. Drugs were either delivered intraperitoneally (i.p.) or locally into PFC and/or NAc via the microdialysis probes. VTA was electrically stimulated with a burst of pulses (pulse width $=1 \mathrm{~ms}$, burst width $=200 \mathrm{~ms}$, interburst interval $(I B I)=500 \mathrm{~ms}$ ) for $20 \mathrm{~min}$. For VTA optogenetic stimulation, blue laser $(\sim 473 \mathrm{~nm})$ pulses were delivered for 20 min using one of two burst protocols: (a) 20 pulses (pulse width $=1 \mathrm{~ms}$ ) at $100 \mathrm{~Hz}$ for $200 \mathrm{~ms}, \mathrm{IBI}=500 \mathrm{~ms}$; and (b) 100 pulses (pulse width $=5 \mathrm{~ms}$ ) at $20 \mathrm{~Hz}$ for $5 \mathrm{~s}, I B I=10 \mathrm{~s}$. In the biochemistry experiments, $1 \mathrm{~mm}$ midbrain slices were treated with drugs, and biotinylation for membrane-bound DAT and Rho activation assays were performed. Midbrain slices were used because of low signal-to-noise ratio, especially for detection of DAT, in the slices from terminal areas. See Supplementary Methods for details.

\section{RESULTS}

Electrical stimulation of VTA produces sustained dopamine release in NAC and PFC

Microdialysis was used to measure $[D A]_{0}$ in NAc and medial prefrontal cortex (mPFC) of freely moving rats during the active phase of their sleep-wake cycle in a home-cage environment. Microdialysis has a limit of detection in the femtomolar range [33], which is $2-3$ orders of magnitude better than the detection limit of FSCV [34]. The improved limit of detection afforded by microdialysis permits measurement of resting baseline $[D A]_{o}$ in behaving animals, including in regions with sparse dopamine innervation such as PFC [22]. We were, therefore, able to detect resting $[D A]_{O}$ in NAC and $\mathrm{MPFC}$ before stimulation of VTA neurons and follow $[D A]_{0}$ until its return to a stable pre-stimulation level.

VTA was electrically stimulated after stable $[D A]_{0}$ was measured in NAc and mPFC. The electrical stimulation protocol $(100 \mathrm{~Hz}$ burst for $200 \mathrm{~ms}, I B I=500 \mathrm{~ms}$ ) mimicked the bursting firing pattern observed in VTA dopamine neurons when rats are engaged in relevant behaviors. For example, in response to reward during a reward-guided operant task, VTA dopamine neurons (identified based on response to apomorphine) fire on average 1.1 bursts per second ( $\mathrm{IBI}=\sim 900 \mathrm{~ms}$ ) with a typical intraburst frequency of $\sim 30 \mathrm{~Hz}$, but they can fire as fast as $100 \mathrm{~Hz}$ [35]. Hence, our protocol utilized interburst and intraburst frequencies that are within the range of reported frequencies. The stimulation duration was 5-20 min to model the typical duration of reward-driven behavioral paradigms $[19,22]$. We found that electrical stimulation of VTA increased $[D A]_{\circ}$ in NAc and $\mathrm{mPFC}$, and these elevations were sustained from several minutes to more than an hour post stimulation (Fig. 1 and S1, S2). VTA stimulation elicited a larger $[D A]_{0}$ increase in PFC compared to NAc (brain region $\times$ sample interaction: $F(9,135)=5.48, p=0.00)$ in the stimulation sample (post hoc independent $t$-test, $p=0.02$ ). $[D A]_{0}$ increases were similar in the post-stimulation samples (sample 5, $p=0.09$; sample $6, p=0.98$; sample 7, $p=0.79$; sample $8, p=0.48$; post hoc $t$ tests). While the difference between PFC and NAc during stimulation is consistent with previous reports of fewer DATs and reduced clearance of extracellular dopamine in PFC $[36,37]$, the present study focused on post-stimulation elevations of $[D A]_{0}$ that were comparable across these regions.

A second stimulation given 140 min later produced a similar pattern of $[D A]_{0}$ increase (Fig. 1), suggesting that the initial stimulation had not provoked a pathological condition, such as sustained depolarization block of dopamine neurons [38]. In addition, the magnitude and duration of dopamine response in both regions were similar to previously reported microdialysis measures in animals performing a reward-guided reversal learning task [22]. These observations indicate that the pattern of stimulation used here is relevant to dopamine cell firing and dopamine release during reward-directed learning and cognitive behaviors. For subsequent studies, we focused on characterizing the impact of 20-min VTA stimulation on [DA] in NAc because of higher dopamine signal in this region compared to PFC (mean baseline concentration in PFC $=0.2 \pm 0.06 \mathrm{fmol}$; in $\mathrm{NAC}=0.8 \pm$ $0.12 \mathrm{fmol})$.

Optogenetic VTA dopamine stimulation produces sustained dopamine release in NAC

In addition to dopamine neurons, VTA contains a significant portion of GABA neurons [39], which could have been activated by VTA electrical stimulation. While it is unlikely that GABA neuron co-activation in the VTA produces terminal release of dopamine, we nonetheless compared dopamine responses in NAc after electrical stimulation of VTA neurons to dopamine responses after selective optogenetic stimulation of VTA dopamine neurons in tyrosine hydroxylase (Th)::Cre rats [40]. We have previously verified highly specific expression of channelrhodopsin-2 (ChR2) in VTA dopamine neurons of this transgenic line [41]. Similar to VTA electrical stimulation, optogenetic stimulation of VTA dopamine neurons elicited prolonged increases in $[D A]_{0}$ in NAc (Fig. 2 and S3). These $[D A]_{0}$ elevations persisted for a shorter duration (post hoc tests yielded significance for up to $20 \mathrm{~min}$ post stimulation) compared to $[D A]_{0}$ increases elicited by VTA electrical stimulation (Fig. 1b). This difference may have resulted from a smaller number of neurons being optogenetically stimulated, since only $\sim 60 \%$ of dopamine neurons co-express ChR2 according to our previous quantification [41].

Post-stimulation sustained [DA] increase in NAc and PFC depends on active release

Next, we assessed whether the observed sustained elevation of post-stimulation $[D A]_{0}$ in $\mathrm{mPFC}$ and NAc resulted from continued active release of dopamine or simply reflected remaining dopamine that was "leftover" after the large burstinduced release. Previous studies have shown that stimulation of dopamine neurons in a burst pattern produces greater release of dopamine than tonic stimulation, even when the number of impulses per second and the total number of impulses are held constant [42, 43]. Thus, burst firing of dopamine neurons can simply produce a large surge of $[D A]_{0}$ that clears away slowly. We found that blockade of voltagegated sodium channels via local application of TTX to either NAC or mPFC immediately after VTA stimulation drastically reduced $[D A]_{0}$ (Fig. 3 and S4). In fact, $[D A]_{0}$ in NAc after TTX application approached the lower limit of detection in post-stimulation samples (mean of sample 6: $20 \pm 7 \%$ and sample 7: $16 \pm 7 \%$ ), whereas stimulation in the absence of TTX continued to produce 
a

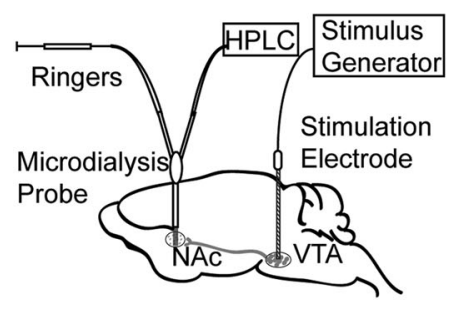

d

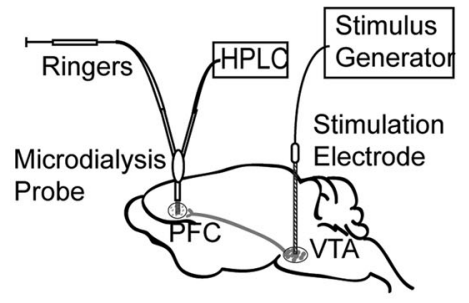

b

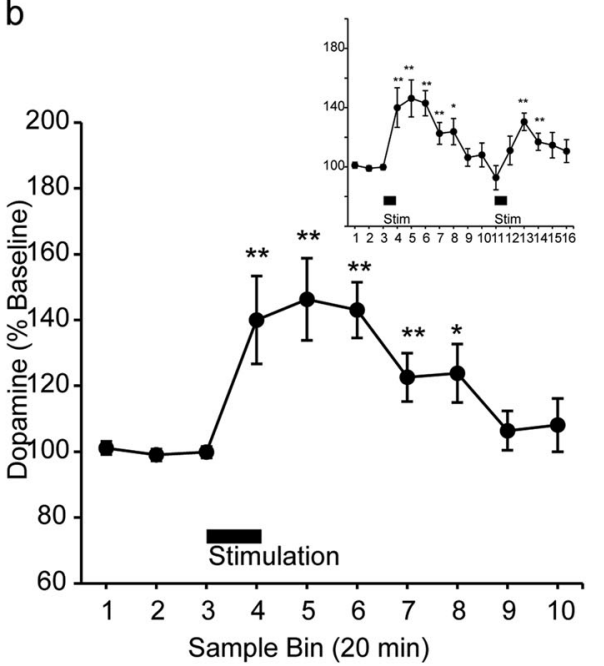

e

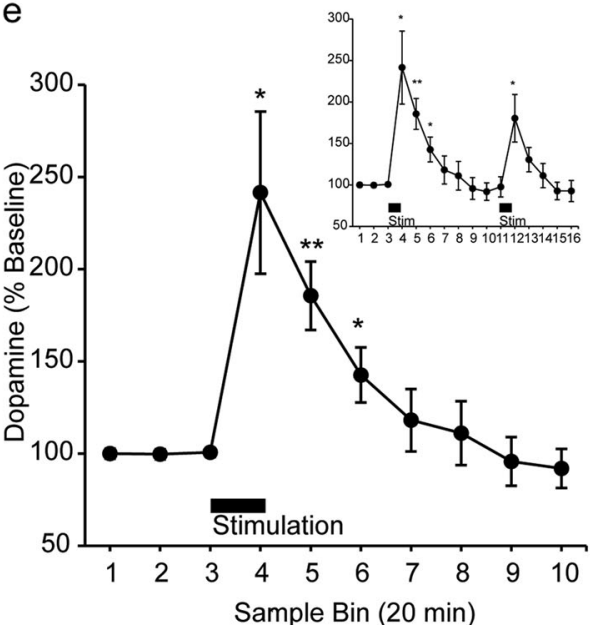

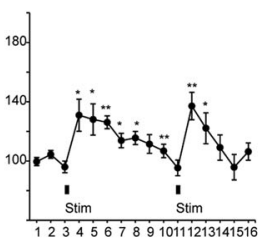

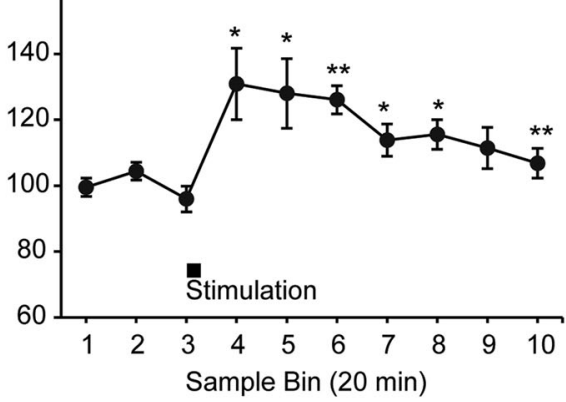

f

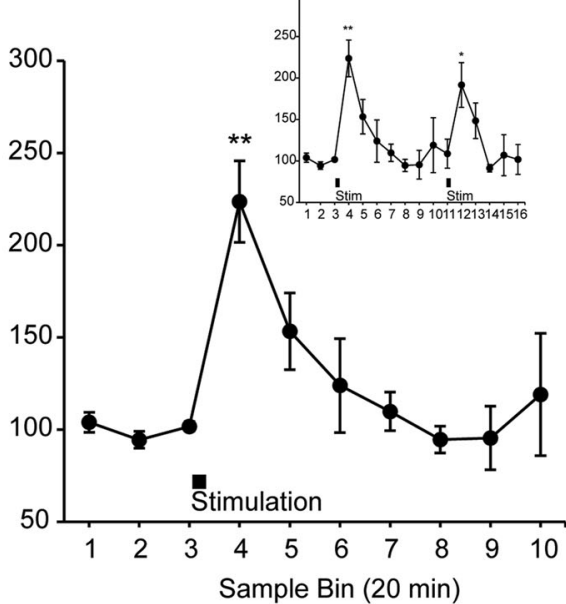

Fig. 1 Electrical stimulation of VTA produces sustained dopamine release in NAC and PFC. a Schematic illustrating simultaneous measurement of $[D A]_{0}$ in NAc via a microdialysis probe and electrical stimulation of VTA via a stimulation electrode. $\mathbf{b}$ VTA was stimulated using a protocol consisting of bursts of $1 \mathrm{~ms}$ pulses delivered at $100 \mathrm{~Hz}$ for $200 \mathrm{~ms}$, with an interburst interval of $500 \mathrm{~ms}$ and an amplitude of $60 \mu \mathrm{A}$, for $20 \mathrm{~min}$ ( $n=10$ rats). VTA activation produced a sustained increase in $[\mathrm{DA}]_{\mathrm{o}}$ in NAc $[\mathrm{F}(15,135)=6.13, p=0.00]$. Post hoc comparisons revealed a significant difference between baseline (sample 3$)$ and sample $4(p=0.01)$, sample $5(p=0.01)$, sample $6(p=0.00)$, sample $7(p=$ $0.01)$, and sample $8(p=0.03)$. The figure inset illustrates dopamine release following a second delivery of $100 \mathrm{~Hz}$ stimulation. The second stimulation also significantly increased $[D A]_{\circ}$ in NAc in samples $13(p=0.00)$ and $14(p=0.01)$. c VTA activation using a stimulus protocol consisting of bursts of $1 \mathrm{~ms}$ pulses delivered at $100 \mathrm{~Hz}$ for $200 \mathrm{~ms}$, with an interburst interval of $500 \mathrm{~ms}$ and an amplitude of $60 \mu \mathrm{A}$ for $5 \mathrm{~min}(n$ $=6$ rats) was associated with a sustained increase in $[D A]_{0}$ in NAc $[F(15,75)=4.93, p=0.00]$. Post hoc comparisons revealed a significant difference between baseline (sample 3$)$ and sample $4(p=0.03)$, sample $5(p=0.02)$, sample $6(p=0.00)$, sample $7(p=0.04)$, sample $8(p=$ $0.02)$, and sample $10(p=0.01)$. The figure inset illustrates dopamine release following a second delivery of $100 \mathrm{~Hz}$ stimulation. The second stimulation resulted in an immediate elevation of $[\mathrm{DA}]_{\mathrm{o}}$ in NAc in sample $12(p=0.01)$ and sample $13(p=0.03)$. Data are represented as mean \pm SEM. ${ }^{*} p \leq 0.05 ;{ }^{* *} p \leq 0.01$. d Schematic illustrating simultaneous measurement of $[D A]_{\circ}$ in PFC via a microdialysis probe and electrical stimulation of VTA via a stimulation electrode. e VTA stimulation using a protocol consisting of bursts of $1 \mathrm{~ms}$ pulses delivered at $100 \mathrm{~Hz}$ for $200 \mathrm{~ms}$, with an interburst interval of $500 \mathrm{~ms}$ and an amplitude of $60 \mu \mathrm{A}$, for $20 \mathrm{~min}\left(n=7\right.$ rats) elevated $[\mathrm{DA}]_{\mathrm{o}}$ in PFC $[\mathrm{F}(15,90)=8.10, p=0.00]$. Post hoc comparisons revealed a significant difference between baseline (sample 3 ) and sample $4(p=0.02)$, sample $5(p=0.01)$ and sample 6 $(p=0.04)$. The inset illustrates dopamine release following a second delivery of $100 \mathrm{~Hz}$ stimulation, which significantly increased [DA] in PFC in sample 12 when compared to baseline $(p=0.04)$. $\mathrm{f}$ VTA activation using a stimulus protocol consisting of bursts of $1 \mathrm{~ms}$ pulses delivered at $100 \mathrm{~Hz}$ for $200 \mathrm{~ms}$, with an interburst interval of $500 \mathrm{~ms}$ and an amplitude of $60 \mu \mathrm{A}$, for 5 min $(n=6$ rats) was associated with sustained elevations in $[D A]_{0}$ in PFC $[F(15,75)=5.15, p=0.00]$. Post hoc comparisons against baseline indicated a significant increase in $[D A]_{0}$ in sample $4(p=0.00)$. The inset illustrates dopamine release following a second delivery of $100 \mathrm{~Hz}$ stimulation. Post hoc comparisons revealed that $[D A]_{0}$ in PFC in sample 12 was significantly increased compared to baseline $(p=0.02)$. Data are represented as mean \pm SEM. ${ }^{*} p \leq 0.05 ;{ }^{* *} p \leq$ 0.01 (See also Fig. S1, S2)

significant elevations in $[D A]_{\circ}$ (mean of sample $6: 143 \pm 8 \%$ and sample 7: $123 \pm 7 \%)$. Similarly, in PFC, mean [DA] after TTX treatment fell to $19 \pm 5 \%$ of baseline by post-stimulation sample 6 while stimulation alone still elicited a significant $[D A]_{0}$ increase at that time point (mean: $143 \pm 15 \%$ ). While some of the reduction in post- $T T X[D A]_{0}$ can be explained by $T T X^{\prime}$ s effect on baseline $[D A]_{0}$, the fact that NAc and PFC $[D A]_{0}$ dropped close to the detection limit suggests that TTX also prevented most of the sustained increase in $[\mathrm{DA}]_{0}$ elicited by VTA stimulation. These results suggest that the sustained post-burst elevation of $[D A]_{0}$ 
a

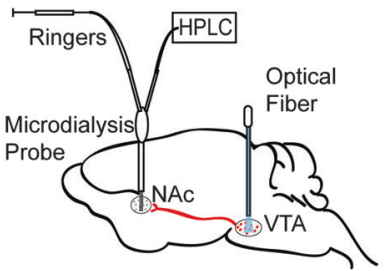

C

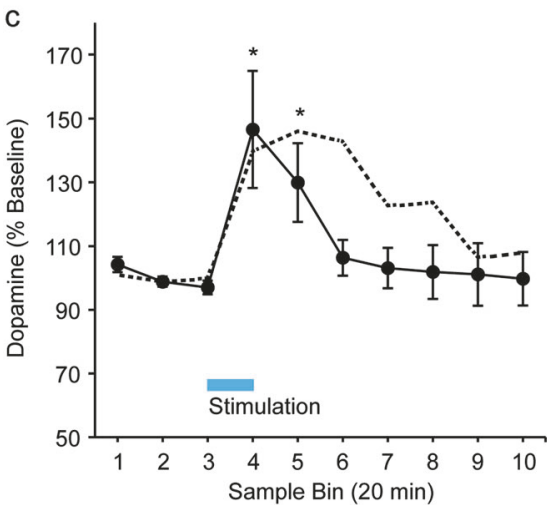

b

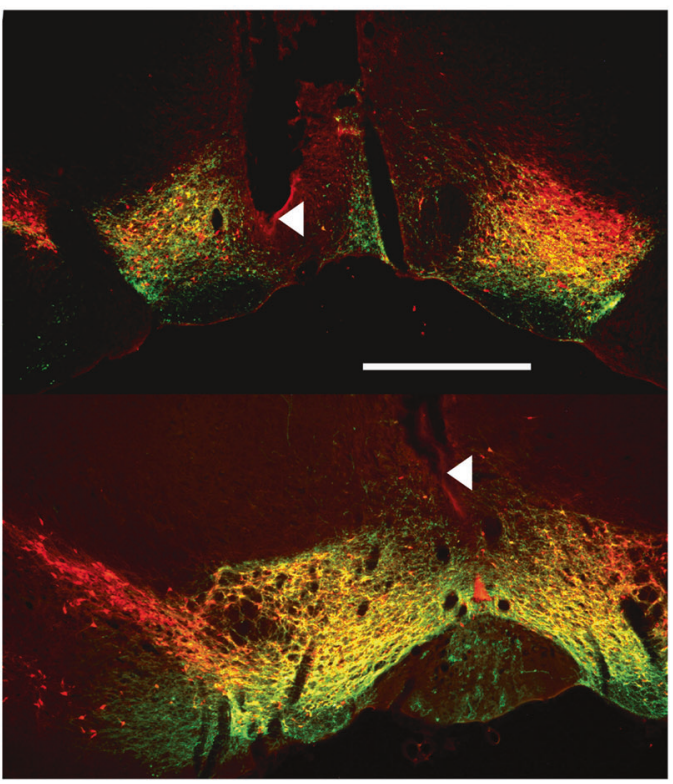

Fig. 2 Optogenetic stimulation of VTA dopamine neurons produces sustained dopamine release in NAc. a Schematic illustrating simultaneous measurement of $[D A]_{0}$ via a microdialysis probe in NAc and blue laser illumination of ChR2-expressing VTA dopamine neurons via an optical fiber. b Immunofluorescence images from two representative Th::Cre rats illustrate expression of ChR2-eYFP (green) in TH+ (red) dopamine neurons in the VTA. Neurons that co-express ChR2 and TH appear yellow. Arrowheads point to optical fiber tips. Scale bar $=1 \mathrm{~mm}$. c VTA dopamine neurons were stimulated using either a protocol consisting of bursts of $1 \mathrm{~ms}$ pulses delivered at $100 \mathrm{~Hz}$ for $200 \mathrm{~ms}$, with an interburst interval of $500 \mathrm{~ms}$, for $20 \mathrm{~min}$ or a protocol consisting of bursts of $5 \mathrm{~ms}$ pulses delivered at $20 \mathrm{~Hz}$ for $5 \mathrm{~s}$, with an interburst interval of $10 \mathrm{~s}$, for $20 \mathrm{~min}$ (laser power $=5-7 \mathrm{~mW})(n=11$ sessions from five rats). Optogenetic VTA dopamine stimulation was associated with a sustained increase in $[D A]_{0}$ in $N A c[F(9,90)=4.47, p=0.00]$. Post hoc comparisons revealed a significant difference between baseline (sample $3)$ and sample $4(p=0.02)$ and sample $5(p=0.02)$. The dotted line represents electrical stimulation-induced elevations in NAc [DA $]_{\mathrm{O}}$ (depicted also in Fig. 1b). Data are represented as mean \pm SEM. ${ }^{*} p \leq 0.05$ (See also Fig. S3)

a

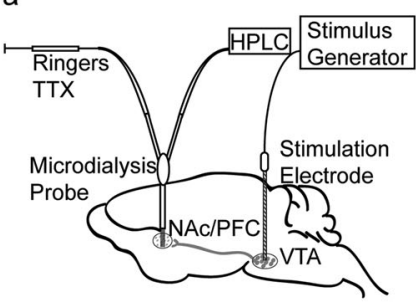

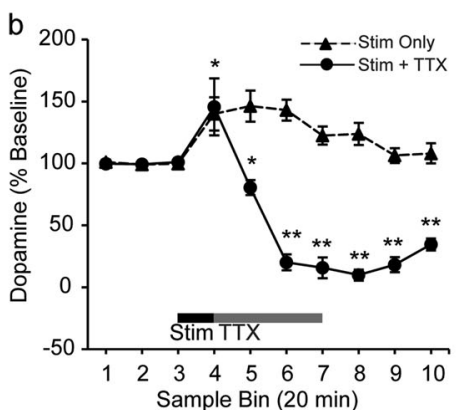

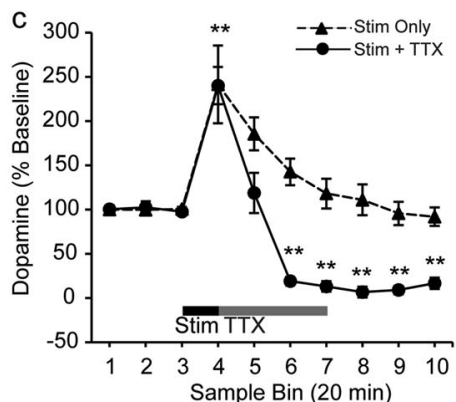

Fig. 3 TTX blocks sustained dopamine release in NAc and PFC. a Schematic of infusion of TTX via microdialysis probes into NAc or PFC after electrical stimulation of VTA. b TTX infusion in NAc after cessation of VTA electrical stimulation blocked the post-stimulation increase in [DA] in NAc. Solid line indicates data from sessions in which TTX infusion in NAc followed electrical stimulation of VTA (stim + TTX, $n=7$ rats) while the dotted line represents data from rats that received electrical stimulation of VTA only (stim only, presented also in Fig. 1b). Stim only and stim + TTX data were analyzed after applying log transformations (see "Supplementary Methods"). Electrical stimulation of VTA alone produced sustained increases in $[D A]_{0}$ (results after data transformation were identical to the results in Fig. 1b; one-way repeated measures ANOVA: $\mathrm{F}(9,81)=5.93, p=0.00$; post hoc tests against baseline: sample $4(p=0.01)$, sample $5(p=0.01)$, sample $6(p=0.00)$, sample $7(p=$ $0.01)$, and sample $8(p=0.04)$ ). TTX treatment modulated stimulation-induced increases in [DA] in NAc (one-way repeated measures ANOVA, $F(9,54)=24.46, p=0.00$ ). Post hoc comparisons against baseline (sample 3 ) indicated that VTA stimulation before TTX infusion in NAc significantly increased NAc [DA] in sample $4(p=0.04)$. Following TTX treatment, [DA] o decreased below baseline in sample $5(p=0.02)$, sample $6(p=0.01)$, sample $7(p=0.00)$, sample $8(p=0.00)$, sample $9(p=0.00)$, and sample $10(p=0.00)$. A two-way repeated measures ANOVA further demonstrated a significant interaction between sample bin and condition (stim $+\mathrm{TTX}$ and stim only) $[\mathrm{F}(9,135)=33.77, p=$ 0.00]. c TTX infusion in PFC after cessation of VTA electrical stimulation blocked the post-stimulation increase in [DA] in PFC. As in $\mathbf{b}$, the solid line indicates stim + TTX condition ( $n=6$ rats) and the dotted line represents electrical stimulation of VTA only (stim only, presented also in Fig. 2b). TTX treatment after VTA electrical stimulation modulated sustained increases in $[D A]_{0}$ (one-way repeated measures ANOVA: $F(9,45)=$ $45.35, p=0.00)$. Post hoc comparisons against baseline (sample 3$)$ indicated that while stimulation increased $[D A]_{\circ}$ in sample $4(p=0.00)$, $[D A]_{\circ}$ was no longer significantly different from baseline in sample $5(p=0.40)$ after TTX infusion in NAc. Further, [DA] in PFC decreased below baseline in the remaining post-stimulation samples $5,6,7,8,9$, and 10 ( $p=0.00$ for all comparisons). A two-way repeated measures ANOVA also demonstrated a significant interaction between sample bin and condition (stim + TTX and stim only) $[F(9,99)=6.79, p=0.00]$. ${ }^{*} p \leq 0.05$ and ${ }^{* *} p \leq 0.01$ for comparison against baseline within the stim + TTX condition only. Data are represented as mean \pm SEM (See also Fig. S4) 
is supported by active (impulse flow-dependent) exocytotic release of dopamine.

Blockade of cannabinoid and nicotinic receptor activity does not impact post-stimulation increase in NAc [DA]

The outcome of the TTX experiment suggested that the burstinduced increase in $[D A]_{0}$ triggers secondary events that enhance active exocytotic dopamine release and/or reduce the clearance of dopamine actively released in the post-stimulation period. In the striatum, several secondary mechanisms that locally regulate release of dopamine have been identified [44, 45]. For example, an increase in synaptic dopamine release and subsequent dopamine receptor stimulation mobilizes the release of endocannabinoids [46] that could activate type- 1 cannabinoid receptors (CB1Rs) on GABA or other neurons that may modulate dopamine release [45]. We found that inhibition of CB1R activity via systemic injection of rimonabant did not change spontaneous $[D A]_{\circ}$ or the pattern of stimulation-induced increase in $[D A]_{0}$ in NAC (Fig. S5).
Another potential mechanism is secondary activation of cholinergic neurons and subsequent stimulation of presynaptic nicotinic acetylcholine receptors ( $\mathrm{nAChRs)}$ on dopamine axons, evoking dopamine release that is independent of dopamine somal activity $[47,48]$. To test this, the effect of local inhibition of nAChRs by mecamylamine on NAC $[D A]_{\text {o }}$ was examined. Our results did not provide support for this mechanism (Fig. S6a,b). Mecamylamine treatment in combination with VTA stimulation also elicited a large variability in $[\mathrm{DA}]_{0}$ in post-stimulation samples, which could be due to the impact of mecamylamine treatment itself on basal $[D A]_{0}$ (Fig. S6c,d).

Post-stimulation sustained increase in $[D A]_{0}$ results from reduced DAT-mediated clearance of dopamine

Next, we considered mechanisms that are involved in the clearance of $[D A]_{0}$. Previously, it has been shown that amphetamine, which acts as a substrate of DAT and causes a large surge of $[D A]_{0}$, induces transient internalization of DAT [49]. Although microscopy studies have not confirmed this internalization [50],
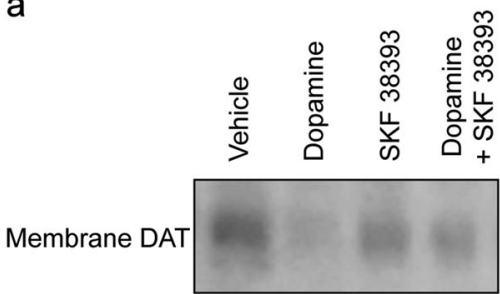

Total DAT
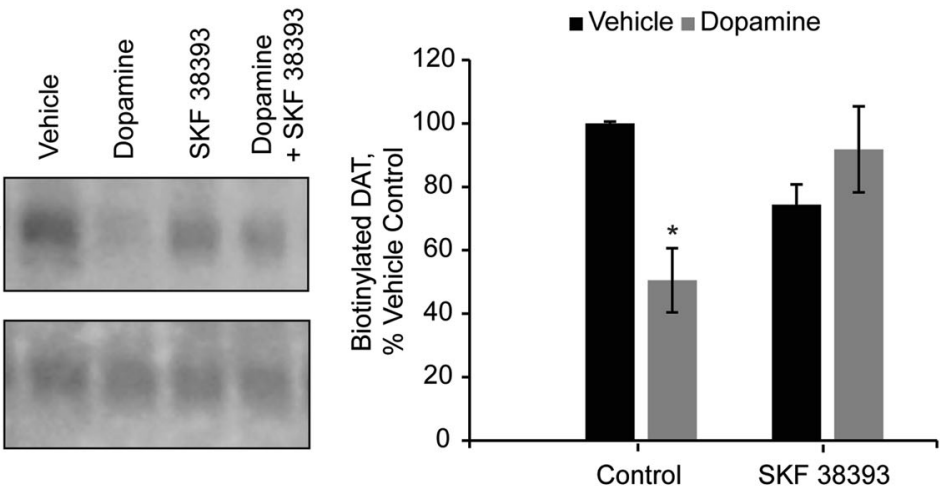

b

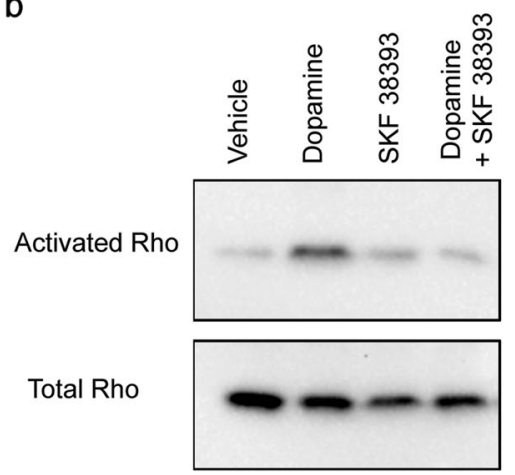

\section{Total DAT}

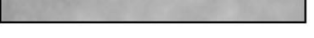

(⿸丆口广

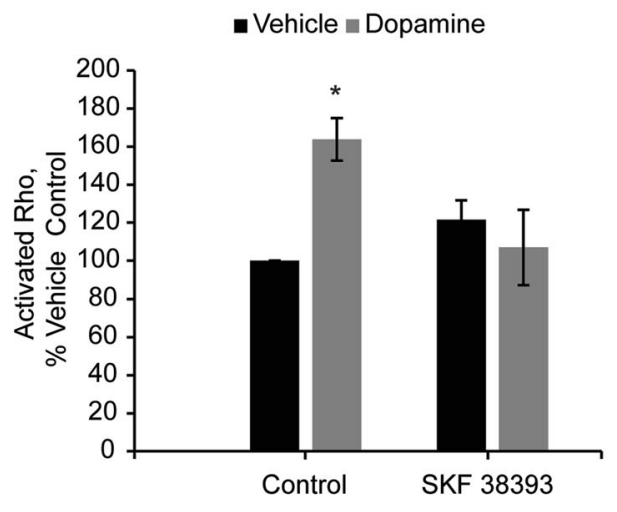

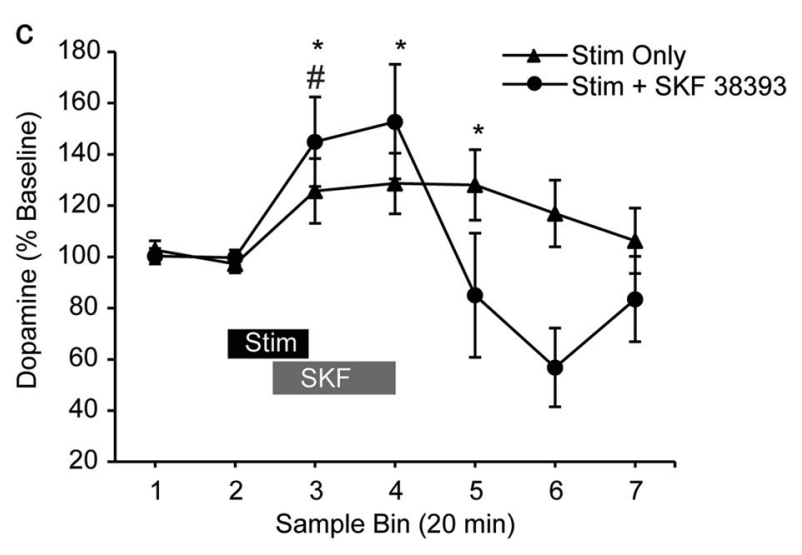


Fig. 4 Stimulation of D1/D5 receptor blocks dopamine-induced decrease in cell surface expression of DAT and increase in Rho activation in vitro as well as attenuates sustained dopamine release in NAc in vivo. a Rat midbrain slices were treated with dopamine (10 $\mu \mathrm{M})$, SKF 38393 $(100 \mathrm{nM})$, or dopamine and SKF 38393. Cell surface proteins were biotinylated, isolated, and probed for cell surface expression. The effect of dopamine on membrane-localized DAT depended on SKF treatment $[F(1,8)=13.72, p=0.01$, dopamine X SKF 38393 interaction, $n=3$ slices for all groups]. Post hoc comparisons against control slices treated with vehicle revealed that membrane-localized DAT was significantly decreased in response to dopamine $(p=0.04)$, but this effect was blocked by SKF $38393(p=2.01)$. SKF 38393 treatment alone did not significantly increase DAT levels but resulted in a non-significant trend toward a decrease in membrane-localized DAT ( $p=0.06)$. $\mathbf{b}$ Tissue lysates were then assessed for activated Rho. A two-way ANOVA revealed a significant interaction between dopamine and SKF 38393 treatments $[\mathrm{F}(1,12)=10.00, p=0.01]$ ( $n=4$ slices all groups). Post hoc comparisons against control slices treated with vehicle indicated that dopamine increased the amount of activated Rho-GTPase in midbrain slices $(p=0.01)$, and this effect was blocked by SKF $38393(p=0.75)$. SKF 38393 alone did not alter the amount of activated Rho-GTPase $(p=0.12) .{ }^{*} p \leq 0.05$. The displayed blot images are cropped from fulllength blots, which are included in Fig. S7. c In vivo treatment of SKF $38393(10 \mu \mathrm{M})$ in NAc 10 min after the start of electrical stimulation of VTA attenuated post-stimulation increase in [DA $]_{0}$ in NAc. Filled triangles represent data from sessions with electrical stimulation of VTA only (stim only, $n=5$ rats) and filled circles represent data from sessions with SKF 38393 infusion in NAc and electrical stimulation of VTA (stim + SKF $38393, n=5$ rats). For these sessions, VTA was stimulated using a protocol consisting of bursts of 1 ms pulses delivered at $100 \mathrm{~Hz}$ for 200 $\mathrm{ms}$, with an interburst interval of $500 \mathrm{~ms}$ and an amplitude of $6 \mu \mathrm{A}$ (see "Supplementary Methods"), for 20 min. Electrical stimulation of VTA increased $[D A]_{0}$ in NAc (one-way repeated measures ANOVA, $\mathrm{F}(6,24)=3.53, p=0.01$ ). Post hoc comparisons against baseline (sample 2) demonstrated significant elevations of [DA $]_{\mathrm{o}}$ in sample $3(p=0.05)$, sample $4(p=0.03)$, and sample $5(p=0.05)$. Electrical stimulation of VTA in combination with SKF 38393 treatment also resulted in a significant modulation of NAc $[D A]_{\circ}$ (one-way repeated measures $A N O V A, F(6,24)=$ $6.29, p=0.00$ ). Post hoc comparisons against baseline (sample 2) demonstrated that even in the presence of SKF 38393 in NAc, electrical stimulation of VTA produced a significant elevation in NAC [DA $]_{0}$ in stimulation sample $3(p=0.04)$ and resulted in a trend toward a significant $[D A]_{0}$ increase in post-stimulation sample $4(p=0.08)$. However, SKF 38393 treatment prevented a sustained stimulation-induced $[D A]_{\circ}$ increase in sample $5(p=0.58)$ and resulted in a trend toward a decrease in [DA] below baseline in sample $6(p=0.06)$. ${ }^{*}$ and \# indicate $p \leq$ 0.05 for comparisons against baseline within stim only and stim + SKF 38393 conditions, respectively. Further, a two-way repeated measures ANOVA confirmed that the local blockade of D1/5 receptors in NAc by SKF 38393 changed the pattern of [DA] increase elicited by electrical stimulation of VTA (sample X condition: $F(6,48)=4.23, p=0.00$ ). Data are represented as mean \pm SEM (See also Fig. S8, S9)

other work indicates that the process is mediated, in part, by activation of Rho-GTPases, because selective inhibition of Rho, or stimulation of protein kinase A (PKA)-coupled receptors such as D1/5 dopamine receptors, prevents amphetamine-induced DAT internalization [51]. We hypothesized that a surge in synaptic dopamine release caused by burst activation of cell bodies also results in DAT internalization. Such a mechanism would then lead to an increase in $[D A]_{0}$ even after cessation of burst activation of dopamine neurons by reducing the rate of clearance of newly released dopamine. As FSCV studies have characterized transient surges in $[D A]_{0}$ in NAc in the range of $1-10 \mu \mathrm{M}$ upon burst dopamine stimulation [10], we applied a comparable concentration of dopamine $(10 \mu \mathrm{M})$ in rat midbrain slices to mimic burstinduced dopamine release in vivo. We found that $10 \mu \mathrm{M}$ dopamine decreased cell surface expression of DATs in midbrain slices (Fig. 4a). The same concentration of dopamine increased the amount of activated Rho-GTPase (Fig. 4b). We then tested the effects of inactivating Rho on dopamine-mediated DAT internalization. To inactivate Rho, we stimulated D1/5 receptors in midbrain slices with SKF 38393. D1/5 receptors are G-proteincoupled receptors (GPCRs) present on dopamine neurons, and stimulation of these GPCRs increases adenylyl cyclase activity that activates PKA [52]. Activated PKA phosphorylates and thus inactivates Rho [53]. We found that SKF 38393 blocked dopamine's effects on Rho activation and DAT internalization (Fig. 4a, b and S7).

To establish the relevance of a Rho-mediated DAT internalization mechanism in freely moving animals, we examined the effect of systemic or intra-NAc application of SKF 38393 on [DA] $]_{0}$ and found that both treatments attenuated sustained increases in $[D A]_{O}$ in post-stimulation samples (Fig. 4c and S8). These slice and in vivo findings, combined with the results from the $\Pi X$ experiment, support the following mechanism for sustained post-stimulation elevation of $[D A]_{0}$ : burst activity of dopamine neurons promotes DAT internalization and thus decreases clearance of dopamine actively released in the post-burst period, leading to a large post-burst $[\mathrm{DA}]_{\mathrm{o}}$ even though dopamine neurons have returned to tonic mode of firing. Consistent with previous work $[54,55]$, SKF 38393 also decreased basal $[D A]_{\circ}$ in NAc (Fig. S9). This suggests that D1/D5 receptor activation may impact DAT internalization in both baseline and stimulation conditions in vivo. Importantly, however, there was no difference in $[D A]_{0}$ between SKF only and SKF + stimulation conditions in post-stimulation samples.

\section{DISCUSSION}

We found that phasic burst activity of VTA dopamine neurons in awake rats in a home-cage environment is followed by a sustained post-burst elevation of $[D A]_{0}$ in both NAC and PFC terminal regions that persisted for at least $20 \mathrm{~min}$. This increase is not due to dopamine spillover from the initial stimulation but depends on continued active release of dopamine in terminal regions. We identified Rho-mediated internalization of DATs as a potential mechanism responsible for increased post-burst availability of actively released dopamine, based on evidence that experimental blockade of Rho activation prevented both DAT internalization and the sustained post-stimulation elevation of $[D A]_{0}$. These results suggest that phasic surges of dopamine release produced by bursting of dopamine neurons result in DAT internalization that reduces the clearance of actively released dopamine in the postburst period, causing a sustained post-burst elevation of tonic $[\mathrm{DA}]_{\mathrm{O}}$.

Sustained post-burst increase in $[D A]_{0}$ is consistent with behavioral evidence and volume transmission

The sustained increase in $[D A]_{0}$ after periods of burst activity is functionally significant, because dopamine perisynaptic architecture in cortical and striatal regions is well suited to accommodate a slow and spatially distributed signal via volume transmission [45, 56]. A considerable density of dopamine receptors, both in the PFC and striatum, is localized extrasynaptically [57-59], and more than half of dopamine release sites in the striatum are reported to lack post-synaptic specializations [60]. Hence, dopamine that slowly accumulates in the extracellular space on the order of minutes to hours could modulate the activity of many pre- and post-synaptic sites within a large area in the striatum and the PFC via volume transmission [28]. This sustained and diffuse dopamine signal might maintain and stabilize/consolidate networks activated during learning and working memory [61]. In addition, 

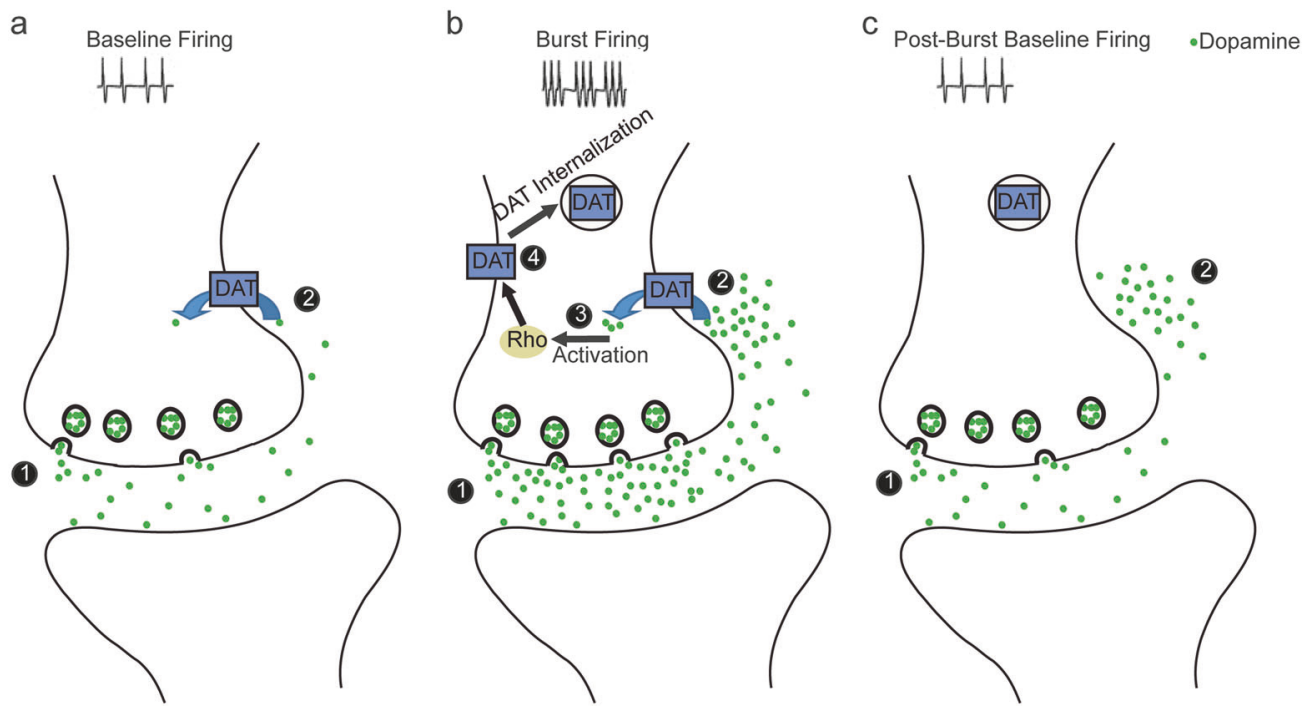

Fig. 5 Mechanism for sustained increase in extracellular dopamine following burst activation of dopamine neurons. a (1) Baseline spontaneous firing of dopamine neurons causes the release of low levels of dopamine from the terminals. (2) Dopamine diffuses into the extrasynaptic space where it is taken up by DATs, resulting in a low level of extracellular dopamine. $\mathbf{b}$ (1) Dopamine neurons fire in phasic bursts in response to behaviorally salient stimuli. Phasic bursting results in increased release and higher synaptic levels of dopamine. (2) A high concentration of extracellular dopamine enters the cell via DATs. (3) Intracellular dopamine activates Rho. (4) Rho activation mediates internalization of DATs. c (1) Upon cessation of phasic bursting, dopamine neurons return to baseline firing and release low levels of dopamine into the synapse. (2) Synaptic dopamine diffuses into the extrasynaptic space, where very few DATs are present following DAT internalization. As a result, extrasynaptic dopamine is cleared away slowly and dopamine accumulates in the extracellular space

persistent dopamine signaling could lower the threshold for longterm potentiation in all synapses for a certain period of time, allowing for adaptive modulation of memory encoding [62].

The mechanisms identified here might be especially critical for explaining dopamine's role in memory consolidation during reinforcement-guided learning. Previous microdialysis studies in animals performing reward-driven learning or working memory tasks have demonstrated that $[D A]_{\circ}$ in PFC and striatal regions remain elevated for many minutes after completion of the training phase [21-23]. In fact, the duration and magnitude of the posttraining increase in $[D A]_{0}$ in these studies were similar to what we observed in the present study after electrical or optogenetic burst stimulation of dopamine neurons. The importance of this posttraining dopamine signaling has been recognized [24-26, 63], but the underlying mechanisms are unknown. Dopamine neurons fire phasically in response to salient stimuli during a behavioral task, but these phasic increases in activity return to baseline levels after hundreds of milliseconds [7, 8, 64-67]. This raises the question of how elevated dopamine levels can be sustained post training, when dopamine neurons are presumably firing at baseline levels. Our data obtained under electrical and optogenetic stimulation conditions suggest that repetitive stimuli-evoked bursting of dopamine neurons during behavioral training may produce changes in DAT internalization processes, resulting in elevated extracellular accumulation of spontaneously released dopamine post training. This finding also reconciles rapid dopaminergic transmission during learning with slow and sustained dopamine response after learning.

Prolonged dopamine responses have been implicated in sustained motivation [6][68-71]. For example, sustained increases in $[D A]_{0}$ that continue after the end of behavior are observed in the striatum when rats lever press for food or lick a spout for sucrose solution, and these prolonged dopamine elevations are modulated by shifts in motivational state $[20,70,72]$. Furthermore, a computational model has indicated that a slow increase in striatal dopamine levels, which might carry an averaged reward signal during a behavioral task, predicts motivational state [73].
Hence, sustained increases in $[D A]_{O}$, as observed in the current study, might be critical for plasticity mechanisms that include memory consolidation and adaptive learning as well as persistent functions such as working memory and motivation.

Proposed mechanism of sustained dopamine release following burst activation

While phasic and sustained (or tonic) modes of dopamine neurotransmission are often hypothesized to be driven by distinct mechanisms and to affect different post-synaptic processes and behaviors [27-29, 74], the present findings suggest that, in awake and active animals, these two modes of signaling may be complementary (Fig. 5). Ongoing activity of dopamine neurons in awake animals maintains impulse flow-dependent resting extracellular levels of dopamine in terminal regions (Fig. 5a) [75]. These levels are regulated by DAT, since the local application of DAT inhibitors profoundly enhances $[D A]_{0}[76,77]$. In response to salient events during behavioral tasks, dopamine neurons respond with brief bursts of spikes $[7,8,64,65,67]$ that generate a large phasic surge of $[D A]_{0}[42,43]$. Our data indicate that large increases in $[D A]_{0}$ may cause DAT internalization via activation of Rho-GTPases. Similar to mechanisms described for amphetamine-mediated internalization of DATs and EAAT3 glutamate transporters on dopamine neurons $[51,78,79]$, we propose that dopamine enters the presynaptic terminal via DATs to initiate a Rho-dependent intracellular signaling cascade (Fig. 5b). The mechanism via which intracellular dopamine activates Rho is unclear. Candidate mechanisms include influx of calcium and subsequent calmodulin/Camkll activation or activation of intracellular GPCRs [79]. DAT internalization has important consequences for the period following burst stimulation, which would be analogous to the immediate post-task period in learning and cognitive tasks. In the post-task period, dopamine neurons return to their spontaneously firing activation level, which generates a low level of synaptic dopamine. However, because of DAT internalization, dopamine is cleared at a much slower rate, leading to a sustained accumulation of dopamine in the extracellular space in terminal regions (Fig. $5 \mathrm{c}$ ). 
Role of D1/5 receptor activation in dopamine-induced DAT internalization

While multiple mechanisms may activate PKA and block Rhomediated DAT internalization, our experimental approach was driven by data showing that D1/5 receptors are physiologically relevant in dopamine-related behaviors. For example, mice lacking D5 receptors display a greater locomotor response to methamphetamine, a dopamine-activating stimulant, compared to wildtype mice [80], suggesting that D5 receptor activation might serve to curb excessive dopamine release and accumulation. Hence, we speculate that when there is a burst-induced release of a large amount of dopamine, activation of D1/5 receptors by extracellular dopamine counteracts, to some extent, Rho activation and DAT internalization mediated by dopamine transported into the cell. In other words, in dopamine terminals at baseline conditions, there might be a balance between processes that promote and interrupt DAT internalization. We speculate that the former could be mediated by intracellular dopamine that activates Rho and/or synaptic dopamine that activates D2 receptors and potentially inhibits PKA activity $[52,81]$ whereas the latter by extracellular dopamine that activates presynaptic D1/5 receptors. When a large surge of dopamine occurs during burst activation of dopamine neurons, the balance might be shifted toward Rho-mediated DAT internalization. Accordingly, when most D1/5 receptors are stimulated by SKF 38393, activation of D1/5-coupled PKA signaling cascades could outcompete ongoing Rho activation. Consistent with this idea, our data indicate that SKF 38393 not only prevents burst-induced increases in $[D A]_{0}$ but also decreases baseline $[D A]_{0}$.

\section{Additional considerations}

The DAT-mediated clearance mechanism we have identified here is likely only one of the mechanisms that contribute to the sustained post-stimulation activation of $[\mathrm{DA}]_{\mathrm{O}}$. Other mechanisms may include activation of feedback loops, such as stimulation of NAc medium spiny neurons that inhibit GABA neurons in VTA, resulting in the disinhibition of VTA dopamine neurons [82], and/ or local signaling via non-dopamine receptors, including glutamate, GABA, and nAChRs [44, 45, 47, 48]. Long-range interactions with the ventral subiculum of the hippocampus can also elicit sustained dopamine release in NAc [83]. Likewise, the sustained elevations of $[D A]_{0}$ observed upon stimulation of the subiculum may be mediated, in part, by the proposed DAT internalization mechanism.

While most experiments in the current study focused on NAC, the proposed Rho-mediated DAT internalization is relevant for sustaining $[D A]_{0}$ elevations in PFC. Although there are fewer DATs in PFC and norepinephrine transporters (NETs) play a significant role in dopamine uptake in this region [84], DAT inhibition significantly reduces $[D A]_{0}$ in PFC [85]. We do not, however, discount the potential involvement of NETs in the observed sustained increase in post-stimulation $[D A]_{0}$.

Although local and systemic application of SKF 38393 elicited similar reduction in post-stimulation $[D A]_{0}$, thus reducing the possibility of the observed effects being mediated by D1/5 receptors at VTA cell bodies, future studies should examine whether local stimulation of D1/5 receptors in terminal regions can indirectly modulate the firing of VTA neurons via long-range loops or can affect dopamine terminal activity through intrastriatal loops mediated by post-synaptic receptors. Finally, future studies should also determine whether burst firing of dopamine neurons at lower frequencies $(15-60 \mathrm{~Hz})$ could result in the observed post-burst-sustained dopamine release via the proposed mechanism.

\section{CONCLUSION}

The present findings indicate that a critical function of burst activity of dopamine neurons may be to produce a persistent elevation of $[D A]_{0}$ in terminal regions via an intracellular mechanism that promotes DAT internalization. The sustained post-burst increase in dopamine levels may be essential for consolidation of memories during associative learning, maintenance of active networks in working memory, and sustenance of motivational states. Hence, identification of a mechanism that supports this persistent increase in $[D A]_{\circ}$ has important implications for understanding learning and memory processes in the brain and may provide insights into dysfunction of dopamine systems in disorders such as ADHD, addiction, and schizophrenia.

\section{ACKNOWLEDGEMENTS}

This work was supported by the National Institutes of Health grant R01 (MH048404) to BM and Intramural Research at the National Institute of Mental Health to SA.

\section{AUTHOR CONTRIBUTIONS}

$A M, A D, S L, B M$, and $M R$ designed and performed the microdialysis experiments. SA and SU designed and performed the biochemistry experiments. LR performed the dual immunofluorescence localizations. AM and SL analyzed the data. BM and SA provided the financial support. SL and BM wrote the paper.

\section{ADDITIONAL INFORMATION}

Supplementary information accompanies this paper at (https://doi.org/10.1038/ s41386-018-0088-7)

Competing interests: The authors declare no competing interests.

Publisher's note: Springer Nature remains neutral with regard to jurisdictional claims in published maps and institutional affiliations.

\section{REFERENCES}

1. Carter RM, Macinnes JJ, Huettel SA, Adcock RA. Activation in the VTA and nucleus accumbens increases in anticipation of both gains and losses. Front Behav Neurosci. 2009;3:21.

2. Baudonnat M, Huber A, David V, Walton ME. Heads for learning, tails for memory: reward, reinforcement and a role of dopamine in determining behavioral relevance across multiple timescales. Front Neurosci. 2013;7:175.

3. Kehagia AA, Murray GK, Robbins TW. Learning and cognitive flexibility: frontostriatal function and monoaminergic modulation. Curr Opin Neurobiol. 2010;20:199-204.

4. Berridge KC. The debate over dopamine's role in reward: the case for incentive salience. Psychopharmacology. 2007;191:391-431.

5. Robbins TW, Arnsten AF. The neuropsychopharmacology of fronto-executive function: monoaminergic modulation. Annu Rev Neurosci. 2009;32:267-87.

6. Salamone JD, Correa M. The mysterious motivational functions of mesolimbic dopamine. Neuron. 2012;76:470-85.

7. Matsumoto $M$, Hikosaka $O$. Two types of dopamine neuron distinctly convey positive and negative motivational signals. Nature. 2009;459:837-41.

8. Schultz W. Predictive reward signal of dopamine neurons. J Neurophysiol. 1998;80:1-27.

9. Garris PA, Ciolkowski EL, Pastore P, Wightman RM. Efflux of dopamine from the synaptic cleft in the nucleus accumbens of the rat brain. J Neurosci. 1994;14:6084-93.

10. Garris PA, Wightman RM. Different kinetics govern dopaminergic transmission in the amygdala, prefrontal cortex, and striatum: an in vivo voltammetric study. J Neurosci. 1994;14:442-50.

11. Lavin A, Nogueira L, Lapish CC, Wightman RM, Phillips PE, Seamans JK. Mesocortical dopamine neurons operate in distinct temporal domains using multimodal signaling. J Neurosci. 2005;25:5013-23.

12. Williams GV, Millar J. Concentration-dependent actions of stimulated dopamine release on neuronal activity in rat striatum. Neuroscience. 1990;39:1-16.

13. Montague PR, Dayan P, Sejnowski TJ. A framework for mesencephalic dopamine systems based on predictive Hebbian learning. J Neurosci. 1996;16:1936-47.

14. Schultz W. Updating dopamine reward signals. Curr Opin Neurobiol. 2013;23:229-38

15. Phillips PE, Stuber GD, Heien ML, Wightman RM, Carelli RM. Subsecond dopamine release promotes cocaine seeking. Nature. 2003;422:614-8. 
16. Stuber GD, Klanker M, de Ridder B, Bowers MS, Joosten RN, Feenstra MG, et al. Reward-predictive cues enhance excitatory synaptic strength onto midbrain dopamine neurons. Science. 2008;321:1690-2.

17. Stuber GD, Roitman MF, Phillips PE, Carelli RM, Wightman RM. Rapid dopamine signaling in the nucleus accumbens during contingent and noncontingent cocaine administration. Neuropsychopharmacology. 2005;30:853-63.

18. Wanat MJ, Willuhn I, Clark JJ, Phillips PE. Phasic dopamine release in appetitive behaviors and drug addiction. Curr Drug Abus Rev. 2009;2:195-213.

19. Cheng JJ, de Bruin JP, Feenstra MG. Dopamine efflux in nucleus accumbens shell and core in response to appetitive classical conditioning. Eur J Neurosci. 2003;18:1306-14.

20. Ostlund SB, Wassum KM, Murphy NP, Balleine BW, Maidment NT. Extracellular dopamine levels in striatal subregions track shifts in motivation and response cost during instrumental conditioning. J Neurosci. 2011;31:200-7.

21. Phillips AG, Ahn S, Floresco SB. Magnitude of dopamine release in medial prefrontal cortex predicts accuracy of memory on a delayed response task. J Neurosci. 2004;24:547-53.

22. Stefani MR, Moghaddam B. Rule learning and reward contingency are associated with dissociable patterns of dopamine activation in the rat prefrontal cortex, nucleus accumbens, and dorsal striatum. J Neurosci. 2006;26:8810-8.

23. van der Meulen JAJ, Joosten RNJMA, de Bruin JPC, Feenstra MGP. Dopamine and noradrenaline efflux in the medial prefrontal cortex during serial reversals and extinction of instrumental goal-directed behavior. Cereb Cortex. 2007;17:1444-53.

24. Dalley JW, Laane K, Theobald DE, Armstrong HC, Corlett PR, Chudasama Y, et al. Time-limited modulation of appetitive Pavlovian memory by D1 and NMDA receptors in the nucleus accumbens. Proc Natl Acad Sci USA. 2005;102:6189-94.

25. Izquierdo LA, Barros DM, da Costa JC, Furini C, Zinn C, Cammarota M, et al. A link between role of two prefrontal areas in immediate memory and in long-term memory consolidation. Neurobiol Learn Mem. 2007;88:160-6.

26. Setlow B, McGaugh JL. Sulpiride infused into the nucleus accumbens posttraining impairs memory of spatial water maze training. Behav Neurosci. 1998;112:603-10.

27. Floresco SB, West AR, Ash B, Moore H, Grace AA. Afferent modulation of dopamine neuron firing differentially regulates tonic and phasic dopamine transmission. Nat Neurosci. 2003;6:968-73.

28. Grace AA, Floresco SB, Goto Y, Lodge DJ. Regulation of firing of dopaminergic neurons and control of goal-directed behaviors. Trends Neurosci. 2007;30:220-7.

29. Bromberg-Martin ES, Matsumoto M, Hikosaka O. Distinct tonic and phasic anticipatory activity in lateral habenula and dopamine neurons. Neuron. 2010;67:144-55.

30. Ikemoto $S$. Dopamine reward circuitry: two projection systems from the ventral midbrain to the nucleus accumbens-olfactory tubercle complex. Brain Res Rev. 2007;56:27-78.

31. Berger B, Gaspar P, Verney C. Dopaminergic innervation of the cerebral cortex: unexpected differences between rodents and primates. Trends Neurosci. 1991;14:21-7.

32. Wise RA. Dopamine, learning and motivation. Nat Rev Neurosci. 2004;5:483-94

33. Moghaddam B, Roth RH, Bunney BS. Characterization of dopamine release in the rat medial prefrontal cortex as assessed by in vivo microdialysis: comparison to the striatum. Neuroscience. 1990;36:669-76.

34. Westerink BHC, Justice JB Jr. Microdialysis compared with other in vivo release models. In: Robinson TE, Justice Jr. JB, editors. Microdialysis in the neurosciences. New York: Elsevier Science Publishers; 1991. p. 23-43.

35. Hyland $\mathrm{BI}$, Reynolds JN, Hay J, Perk CG, Miller R. Firing modes of midbrain dopamine cells in the freely moving rat. Neuroscience. 2002;114:475-92.

36. Cass WA, Gerhardt GA. In vivo assessment of dopamine uptake in rat medial prefrontal cortex: comparison with dorsal striatum and nucleus accumbens. J Neurochem. 1995;65:201-7.

37. Sesack SR, Hawrylak VA, Matus C, Guido MA, Levey Al. Dopamine axon varicosities in the prelimbic division of the rat prefrontal cortex exhibit sparse immunoreactivity for the dopamine transporter. J Neurosci. 1998;18:2697-708.

38. Grace AA, Bunney BS. Induction of depolarization block in midbrain dopamine neurons by repeated administration of haloperidol: analysis using in vivo intracellular recording. J Pharmacol Exp Ther. 1986;238:1092-1100.

39. Nair-Roberts RG, Chatelain-Badie SD, Benson E, White-Cooper H, Bolam JP Ungless MA. Stereological estimates of dopaminergic, GABAergic and glutamatergic neurons in the ventral tegmental area, substantia nigra and retrorubral field in the rat. Neuroscience. 2008;152:1024-31.

40. Witten IB, Steinberg EE, Lee SY, Davidson TJ, Zalocusky KA, Brodsky M, et al. Recombinase-driver rat lines: tools, techniques, and optogenetic application to dopamine-mediated reinforcement. Neuron. 2011;72:721-33.

41. Lohani S, Poplawsky AJ, Kim SG, Moghaddam B. Unexpected global impact of VTA dopamine neuron activation as measured by opto-fMRI. Mol Psychiatry. 2017;22:585-94.
42. Gonon FG. Nonlinear relationship between impulse flow and dopamine released by rat midbrain dopaminergic neurons as studied by in vivo electrochemistry. Neuroscience. 1988;24:19-28.

43. Bean AJ, Roth RH. Extracellular dopamine and neurotensin in rat prefrontal cortex in vivo: effects of median forebrain bundle stimulation frequency, stimulation pattern, and dopamine autoreceptors. J Neurosci. 1991;11:2694-702.

44. Cachope R, Cheer JF. Local control of striatal dopamine release. Front Behav Neurosci. 2014;8:188

45. Rice ME, Patel JC, Cragg SJ. Dopamine release in the basal ganglia. Neuroscience. 2011;198:112-37.

46. Giuffrida A, Parsons LH, Kerr TM, Rodriguez de Fonseca F, Navarro M, Piomelli D. Dopamine activation of endogenous cannabinoid signaling in dorsal striatum. Nat Neurosci. 1999:2:358-63.

47. Threlfell S, Cragg SJ. Dopamine signaling in dorsal versus ventral striatum: the dynamic role of cholinergic interneurons. Front Syst Neurosci. 2011;5:11.

48. Threlfell S, Lalic T, Platt NJ, Jennings KA, Deisseroth K, Cragg SJ. Striatal dopamine release is triggered by synchronized activity in cholinergic interneurons. Neuron. 2012;75:58-64.

49. Saunders C, Ferrer JV, Shi L, Chen J, Merrill G, Lamb ME, et al. Amphetamineinduced loss of human dopamine transporter activity: an internalizationdependent and cocaine-sensitive mechanism. Proc Natl Acad Sci USA 2000;97:6850-5.

50. Block ER, Nuttle J, Balcita-Pedicino JJ, Caltagarone J, Watkins SC, Sesack SR, et al Brain region-specific trafficking of the dopamine transporter. J Neurosci. 2015;35:12845-58.

51. Wheeler DS, Underhill SM, Stolz DB, Murdoch GH, Thiels E, Romero G, et al. Amphetamine activates Rho-GTPase signaling to mediate dopamine transporter internalization and acute behavioral effects of amphetamine. Proc Natl Acad Sci USA. 2015;121:E7138-47.

52. Neve KA, Seamans JK, Trantham-Davidson H. Dopamine receptor signaling. J Recept Signal Transduct Res. 2004;24:165-205.

53. Ellerbroek SM, Wennerberg K, Burridge K. Serine phosphorylation negatively regulates RhoA in vivo. J Biol Chem. 2003;278:19023-31.

54. Imperato A, Di Chiara G. Effects of locally applied D-1 and D-2 receptor agonists and antagonists studied with brain dialysis. Eur J Pharmacol. 1988;156:385-93.

55. Zetterstrom T, Sharp T, Ungerstedt U. Effect of dopamine D-1 and D-2 receptor selective drugs on dopamine release and metabolism in rat striatum in vivo. Naunyn Schmiede Arch Pharmacol. 1986;334:117-24.

56. Rice ME, Cragg SJ. Dopamine spillover after quantal release: rethinking dopamine transmission in the nigrostriatal pathway. Brain Res Rev. 2008;58:303-13.

57. Caille I, Dumartin B, Bloch B. Ultrastructural localization of D1 dopamine receptor immunoreactivity in rat striatonigral neurons and its relation with dopaminergic innervation. Brain Res. 1996;730:17-31.

58. Lapish CC, Kroener S, Durstewitz D, Lavin A, Seamans JK. The ability of the mesocortical dopamine system to operate in distinct temporal modes. Psychopharmacology. 2007;191:609-25.

59. Smiley JF, Levey Al, Ciliax BJ, Goldman-Rakic PS. D1 dopamine receptor immunoreactivity in human and monkey cerebral cortex: predominant and extrasynaptic localization in dendritic spines. Neurobiology. 1994;91:5720-4.

60. Descarries L, Watkins KC, Garcia S, Bosler O, Doucet G. Dual character, asynaptic and synaptic, of the dopamine innervation in adult rat neostriatum: a quantitative autoradiographic and immunocytochemical analysis. J Comp Neurol. 1996;375:167-86

61. Durstewitz D, Seamans JK, Sejnowski TJ. Dopamine-mediated stabilization of delay-period activity in a network model of prefrontal cortex. J Neurophysiol. 2000;83:1733-50.

62. Shohamy D, Adcock RA. Dopamine and adaptive memory. Trends Cogn Sci. 2010;14:464-72

63. Packard MG, White NM. Memory facilitation produced by dopamine agonists: role of receptor subtype and mnemonic requirements. Pharmacol Biochem Behav. 1989;33:511-8.

64. Pan WX, Schmidt R, Wickens JR, Hyland BI. Dopamine cells respond to predicted events during classical conditioning: evidence for eligibility traces in the rewardlearning network. J Neurosci. 2005;25:6235-42.

65. Roesch MR, Calu DJ, Schoenbaum G. Dopamine neurons encode the better option in rats deciding between differently delayed or sized rewards. Nat Neurosci. 2007;10:1615-24.

66. Schultz W, Apicella P, Ljungberg T. Responses of monkey dopamine neurons to reward and conditioned stimuli during successive steps of learning a delayed response task. J Neurosci. 1993;13:900-13.

67. Takahashi YK, Roesch MR, Wilson RC, Toreson K, O'Donnell P, Niv Y, et al Expectancy-related changes in firing of dopamine neurons depend on orbitofrontal cortex. Nat Neurosci. 2011;14:1590-7.

68. Hauber W. Dopamine release in the prefrontal cortex and striatum: temporal and behavioural aspects. Pharmacopsychiatry. 2010(Suppl 1):43:S32-41. 
69. Howe MW, Tierney PL, Sandberg SG, Phillips PE, Graybiel AM. Prolonged dopamine signalling in striatum signals proximity and value of distant rewards. Nature. 2013;500:575-9.

70. Phillips AG, Vacca G, Ahn S. A top-down perspective on dopamine, motivation and memory. Pharmacol Biochem Behav. 2008;90:236-49.

71. Braver TS, Krug MK, Chiew KS, Kool W, Westbrook JA, Clement NJ, et al. Mechanisms of motivation-cognition interaction: challenges and opportunities. Cogn Affect Behav Neurosci. 2014;14:443-72.

72. Genn RF, Ahn S, Phillips AG. Attenuated dopamine efflux in the rat nucleus accumbens during successive negative contrast. Behav Neurosci. 2004;118:869-73.

73. Niv Y, Daw ND, Joel D, Dayan P. Tonic dopamine: opportunity costs and the control of response vigor. Psychopharmacology. 2007;191:507-20.

74. Cohen JD, Braver TS, Brown JW. Computational perspectives on dopamine function in prefrontal cortex. Curr Opin Neurobiol. 2002;12:223-9.

75. Takahata R, Moghaddam B. Glutamatergic regulation of basal and stimulusactivated dopamine release in the prefrontal cortex. J Neurochem. 1998;71:1443-9.

76. Adams BW, Bradberry CW, Moghaddam B. NMDA antagonist effects on striatal dopamine release: microdialysis studies in awake monkeys. Synapse. 2002;43:12-8.

77. Carboni E, Imperato A, Perezzani L, Di Chiara G. Amphetamine, cocaine, phencyclidine and nomifensine increase extracellular dopamine concentrations preferentially in the nucleus accumbens of freely moving rats. Neuroscience. 1989;28:653-61.
78. Kahlig KM, Lute BJ, Wei Y, Loland CJ, Gether U, Javitch JA, et al. Regulation of dopamine transporter trafficking by intracellular amphetamine. Mol Pharmacol. 2006;70:542-8.

79. Underhill SM, Wheeler DS, Li M, Watts SD, Ingram SL, Amara SG. Amphetamine modulates excitatory neurotransmission through endocytosis of the glutamate transporter EAAT3 in dopamine neurons. Neuron. 2014;83:404-16.

80. Hayashizaki S, Hirai S, Ito Y, Honda Y, Arime Y, Sora I, et al. Methamphetamine increases locomotion and dopamine transporter activity in dopamine d5 receptor-deficient mice. PLoS ONE. 2013;8:e75975.

81. Grace AA, Floresco SB, Goto Y, Lodge DJ. Regulation of firing of dopaminergic neurons and control of goal-directed behaviors. Trends Neurosci. 2007;30:220-7.

82. Xia Y, Driscoll JR, Wilbrecht L, Margolis EB, Fields HL, Hjelmstad GO. Nucleus accumbens medium spiny neurons target non-dopaminergic neurons in the ventral tegmental area. J Neurosci. 2011;31:7811-6.

83. Blaha CD, Yang CR, Floresco SB, Barr AM, Phillips AG. Stimulation of the ventral subiculum of the hippocampus evokes glutamate receptor-mediated changes in dopamine efflux in the rat nucleus accumbens. Eur J Neurosci. 1997;9:902-11.

84. Carboni E, Tanda GL, Frau R, Di Chiara G. Blockade of the noradrenaline carrier increases extracellular dopamine concentrations in the prefrontal cortex: evidence that dopamine is taken up in vivo by noradrenergic terminals. J Neurochem. 1990;55:1067-70.

85. Mundorf ML, Joseph JD, Austin CM, Caron MG, Wightman RM. Catecholamine release and uptake in the mouse prefrontal cortex. J Neurochem. 2001;79:130-42. 